\title{
An Improvement in Compliance for Foot Care in Persons with Type 2 Diabetes with a Teaching Session
}

Satya Ranjan Patra ${ }^{1 *}$ and G. Jahnavi ${ }^{2}$

${ }^{1}$ Associate Professor, Department of General Surgery, Madha Medical College, Thandlam ,Kovur (Near Porur), District -Kancheepuram Chennai 600122 (Tamil Nadu) India

${ }^{2}$ Professor, Department of Community Medicine, Madha Medical College, Thandlam, Kovur (Near Porur), District -Kancheepuram Chennai 600122 (Tamil Nadu) India

\begin{abstract}
Background: Is teaching material actually effective in imparting knowledge and molding attitude? The objective of the study was to implement an educational program in our rural health center Veleru and to evaluate its effect on the foot care of the diabetes patients. This study was conducted to assess the compliance of patients after educating them about foot care.
\end{abstract}

Methods: Improvement in knowledge about foot care and the compliance in the patient population were studied by giving a questionnaire before the teaching session after the teaching session and at one month follow up in 40 type2 diabetic patients residing in the catchment area of the rural health center Veleru. Ninety three percent of the patients did not have any knowledge regarding foot care prior to the education session.

Results: Immediately after the education session $93 \%$ of the patients could answer correctly and after 1 month follow up it was seen that $57 \%$ of all the patients had incorporated the recommendations for foot care.

Conclusion: All diabetic patients if given proper education and guidance regarding foot care would be able to make a significant improvement in their life style which is helpful to avoid amputations.

Keywords: Type2 diabetes; Compliance; Foot care; Teaching session

\section{Introduction}

Though diabetes is an urban problem it is increasing in rural areas also now a days because of changing lifestyles. The prevalence of diabetes is increasing in our rural health center area Veleru. Veleru is a primarily agricultural village having a population of 5000 . The population there had access to clean drinking water and proper sanitation. We wanted to educate people there so we have chosen our rural health center attached to our medical college for our study. Better health care lead to longevity and improvement in technology ensured less physical strain on humans; but it also ensured lifestyle diseases like obesity and diabetes. Diabetes is expected to rise by $42 \%$ in developed and about 200\%in developing nations; further it is expected to increase by three times in Asia with major contribution s from India and China ${ }^{1}$. It is expected to increase from 31.7 million to 79.4 million in India and from 20.8 to 42.3 million in China in a span of 30 years from 2000 to 2030 [1]. Further while persons with diabetes mellitus are more in developed countries are mostly elderly, most of those in developing countries are younger (45-64), thus increasing the impact of diabetes mellitus on those populations and societies [1]

Outcome of diabetes treatment is confounded by patient's ignorance about the disease in general and complete ignorance about its various manifestations in particular. Treatment of diabetes needs patient's participation and a willingness to understand the complex nature of the disease and willpower to withstand a life time of very strict diet exercise and personal care regimen [2].

Diabetes ranks amongst the top indications for non traumatic lower limb amputation. Most diabetic patients visit surgical outpatient department for foot lesions. Fortunately most can avoid by being more caring for their feet. All patients visiting hospital should have their feet_examined routinely for minor lesions like corns and callosity. Approximately $15 \%$ of all patients with Diabetes Mellitus are likely to suffer from foot ulcer at some point of time. Most foot ulcers eventually lead to amputation [3]. Eighty four percent of foot ulcers are attributed to improper foot wear, neglected paronychia or minor mechanical trauma [4]. De Berardis et al opined that people who have been educated about foot care are more likely to examine their feet regularly [5].

In most clinics in our country there is neither time nor resources to devote to individual foot care demonstrations. We were interested to emphasize the importance of foot care to our diabetic patients in Veleru. We developed teaching material like charts and video clips based on cognitive learning theory to educate patients and families on the various aspects of foot care. The content of the teaching session was based on current best practice guidelines [6,7]. This study was conducted to assess the effectiveness in changing patient's perceptions of the importance of proper foot care.

\section{Methods}

The study was conducted at the Rural Health Center Veleru, attached to our college Pinnameneni Siddartha Institute of Medical Sciences Krishna District Andhra Pradesh India; from November 2009 to January 2010. Male and female patients aged between 18-69 years who were already diagnosed as type 2 diabetes and were on treatment

*Corresponding author: Dr. Satya Ranjan Patra, Associate Professor, Dept. of General Surgery, Madha Medical College, Thandlam, Kovur (Near Porur), District -Kancheepuram Chennai 600122 (Tamil Nadu) India, Tel: 91 9963503735; Fax 914424780798 (o); E-mail: patrasatyaranjan@rediffmail.com

Received April 26, 2011; Accepted May 18, 2011; Published May 19, 2011

Citation: Patra SR, Jahnavi G (2011) An Improvement in Compliance for Foot Care in Persons with Type 2 Diabetes with a Teaching Session. J Diabetes Metab 2:130. doi:10.4172/2155-6156.1000130

Copyright: (C) 2011 Patra SR, et al. This is an open-access article distributed unde the terms of the Creative Commons Attribution License, which permits unrestricted use, distribution, and reproduction in any medium, provided the original author and source are credited. 
residing in Veleru were instructed to assemble in the rural health center Veleru on world diabetes day which is observed on the $14^{\text {th }}$ of November. Exclusion criteria were patients residing outside the catchment area because it would be difficult for follow up, visual or hearing impaired that would preclude their viewing the teaching material and those who were not willing to participate in the study.

A total of 40 patients fulfilling the criteria were first assembled in the demonstration room of our health center. They were introduced, their consent was taken and then a questionnaire was given to them which was a pre test.

At first the patients were introduced to the study team. We imparted them with information about type 2 diabetes mellitus and extolled them to participate actively, supporting members of the family like wife and kids were also involved.

Then the actual teaching session consisting of 10 short video clips, 20 flip charts regarding foot care, a set of 50 photographs of different food stuffs representing 100 calories each was shown to all the patients and the main topics discussed were on foot care, regular physical activity and diet. We helped each patient to design a meal plan of $1000 \mathrm{cal} /$ day by showing them a plate with various food stuffs of $100 \mathrm{cal}$ each. They were asked to strictly follow this meal every day. Individual attention was given to each patient and their doubts were clarified, their feet were examined and log books were given to them for recording body weight, blood glucose levels, and care of their feet.

Compliance and implementation of the knowledge gained after the teaching session was tested by filling up of questionnaire before the session, after the session; and at one month follow up. The questions were designed in local language to check if they understood and followed the key points told to them.

The post-test questionnaire was administered after the participants viewed the teaching material and after clarification of their doubts. The participants were asked to come back after a month to fill up the follow up questionnaire. The participants were coded and a separate envelope was kept for each of them so that all the 3 questionnaires of each participant would be maintained separately.

Same set of questionnaire was administered each time. Five questions were designed to test their knowledge about the disease in general, four questions were about foot care in particular; of which two were focused upon the qualitative nature of study, like their pre education knowledge, the improvement in knowledge, incorporation of the teaching in their day to day activities, changes if any in their life style, and any of it is adequate to their needs or if they wanted further teaching sessions.

\section{Results}

\section{Patients}

A total of 46 diabetic patients of type 2 residing in the catchment area of our Rural Health Center Veleru were approached to participate in the study. Patients who were not interested to come back after one month for follow up and those who were visual or hearing impaired were excluded from the study. Thus 40 consented to participate, with all of them returning after 1 month to fill up the follow up questionnaire.

\section{Knowledge about Diabetes Mellitus}

For each of the five questions based on self knowledge about Diabetes Mellitus, there was a significant improvement in the level of knowledge after attending the teaching session that was maintained or surpassed at the one-month follow up. Table 1 demonstrates the percentage of patients self knowledge at each survey point.

\section{Foot-care knowledge}

For the four specific foot-care knowledge questions, Table 2 demonstrates the increase in knowledge that was largely maintained at one-month follow up.

\section{Education received and perceived adherence}

On follow up after one month, 57\% of the patients mentioned that they had followed and practiced what was taught to them. The distribution of responses is demonstrated in Figure 1.

\section{Discussion}

People with diabetes and their families self selected for the teaching session, therefore they were the more motivated ones to take care of themselves, possibly the more socially advantaged. Out of 46 patients who were selected for the study 2 were deaf mute, 1 was visually handicapped and 3 of them said they cannot come for the 1 month follow up, so a total of 40 patients (86.95\%) were included in the study. We could not motivate (6.9\%) of the known cases to attend the teaching session.

It is alarming to find that as many as $93 \%$ of the patients were ignorant about foot care before attending the teaching session. It implies that the patients are not being told about the importance of foot care at the time of diagnosis or on follow up.

\begin{tabular}{|c|c|c|c|}
\hline Awareness of patients & $\begin{array}{l}\text { No. of patients with correct } \\
\text { answer pre-test }(n=40)\end{array}$ & $\begin{array}{l}\text { No. of patients with correct } \\
\text { answer post-test }(n=40)\end{array}$ & $\begin{array}{l}\text { No. of patients with correct answer } \\
\text { one-month follow up }(n=40)\end{array}$ \\
\hline Knowledge of foot care & $3(7.5 \%)$ & $32(82.5 \%)$ & $30(75 \%)$ \\
\hline Knowledge of the importance to check the inside of socks and shoes & $3(7.5 \%)$ & $34(85 \%)$ & $37(92.5 \%)$ \\
\hline Knowledge of importance to always wear foot wear & $17(42.5 \%)$ & $37(92.5 \%)$ & $39(97.5 \%)$ \\
\hline Knowledge of the importance to wear properly fitted shoes & $4(10.0 \%)$ & $34(85 \%)$ & $31(77.5 \%)$ \\
\hline Knowledge about importance of not removing corns or callus by self & $11(27.5 \%)$ & $31(77.5 \%)$ & $39(97.5 \%)$ \\
\hline
\end{tabular}

Table 1: Changes in patient's awareness before, after, and upon follow-up.

\begin{tabular}{|l|l|l|l|}
\hline Knowledge of patients & $\begin{array}{l}\text { No.\& Percentage of patients with } \\
\text { correct answer pre-test }(\mathrm{n}=40)\end{array}$ & $\begin{array}{l}\text { No.\& Percentage of patients with } \\
\text { correct answer post-test }(\mathrm{n}=40)\end{array}$ & $\begin{array}{l}\text { No.\& Percentage of patients with correct } \\
\text { answer after one-month follow up }(\mathrm{n}=40)\end{array}$ \\
\hline What would you do if you found ulcer on your foot & $13(32.5 \%)$ & $37(92.5 \%)$ & $38(95 \%)$ \\
\hline How often do you examine your feet & $10(25 \%)$ & $34(85 \%)$ & $35(87.5 \%)$ \\
\hline What should you use to wash your feet & $30(75 \%)$ & $38(95 \%)$ & $38(95 \%)$ \\
\hline What is the best way to examine the skin of your feet & $5(12.5 \%)$ & $33(82.5 \%)$ & $30(75 \%)$ \\
\hline
\end{tabular}

Table 2: Changes in patient's knowledge before, after, and upon follow up. 


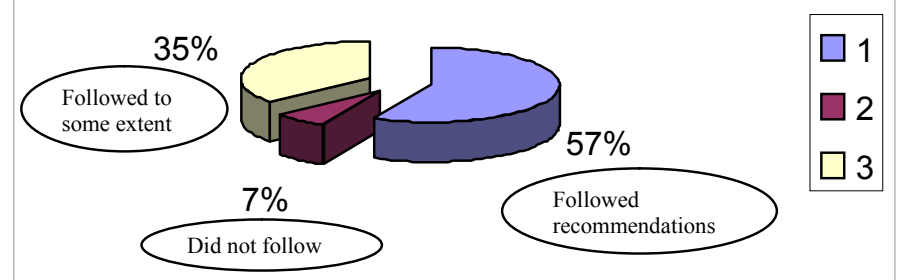

Figure 1: Perceived adherence to recommendations for Foot care after one month follow up.

Table 1 shows a dramatic increase in the knowledge of foot care after educating the patients. In all the five questions on knowledge about diabetes mellitus there was a positive improvement in scores at the post-test compared with the pre-test. At one month follow up the patients had shown improvement in all the five questions based on actual foot care knowledge (Table 2). This shows that the teaching had improved in raising the awareness of the patients about foot care as they had incorporated proper foot care knowledge in their day to day life which is proved by the fact that they are choosing the correct answer in all the questions based on foot care knowledge.

We administered the same set of questionnaire at one month follow up to test how much information was retained; and not surprisingly it was very high. It is well known that recently acquired education is likely to be forgotten unless efforts are made to implement it regularly. In our study a large majority that is above $90 \%$ of the patients had mentioned that they were practicing what had been taught to them (Figure 1).

Most of the patients were daily wage workers who work in the fields. The proper diabetic pressure off loading foot wear is expensive; costing around Rs1000 (20\$). As an alternative in India foot wear made up of MicroCellularRubber is recommended which is equally good and are not expensive; costing around Rs250 (5\$). Although all the patients who had attended the teaching session had purchased the foot wear, yet a few $(7 \%)$ preferred to walk bare feet.

\section{Conclusions}

This study shows that educating the patients about foot care helps them in understanding the problems that can occur if they do not take care of their feet. The results also show that majority of the patients had assimilated and incorporated the information given to them through the teaching material shown and explained to them immediately as well as after one month follow up. Most patients responded that they were benefited by the teaching given to them.

All diabetic patients if given proper education and guidance regarding foot care would be able to make a significant improvement in their life style which is helpful to avoid amputations.

\section{Recommendations}

All people with diabetes should receive basic foot care education, and frequent examination of the foot by the multipurpose health workers and the patients themselves will prevent the complications of feet.

\section{References}

1. Prevention of blindness from diabetes mellitus A report of WHO consultation in Geneva, Switzerland, 9-11 November 2005.

2. Gagliardino JJ, Etchegoyen G, PENDID-LA Research Group (2001) A Mode Educational Program for People With Type 2 Diabetes. Diabetes Care 24: 1001-1007.

3. Gibbons GW, Eliopoulos GM, Infection of the diabetic foot in Kozak GP, Campbell DR, Fryberg RG, Habershaw GM, (eds.). Management of Diabetic Foot Problems, Second edition, Philadelphia WB Saunders 1995: 121.

4. Apelqvisg J, Larsson J, Agardh CD (1990) The influence of external precipitating factors and peripheral neuropathy on the development and outcome of diabetic foot ulcers. J Diabet Complications 4: 21-25.

5. De Berardis G, Pellegrini F, Franciosi M, Belfiglio M, Di Nardo B, et al. (2004) physician attitudes toward foot care education and foot care examination and their correlation with patient practice. Diabetes Care 27: 286-287.

6. Inlow S, Orsted H, Sibbald G (2000) Best practices for the prevention, diagnosis and treatment of diabetic foot ulcers. Ostomy Wound Manage 46: 55-68.

7. Wunderlich RP, Armstrong DG, Hussain SK, Lavery LA (1998) Defining loss of protective sensation in sensation in the diabetic foot. Adv Wound Care 11: 123-128. 